\title{
Linx
}

Revue des linguistes de l'université Paris X Nanterre

39 | 1998

Modèles linguistiques : convergences, divergences

\section{Benoît Habert, Adeline Nazarenko, André Salem, Les linguistiques de corpus}

Armand Colin, Paris, 1997

\section{OpenEdition \\ Journals}

Édition électronique

URL : http://journals.openedition.org/linx/933

DOI : $10.4000 /$ linx.933

ISSN : 2118-9692

Éditeur

Presses universitaires de Paris Nanterre

Édition imprimée

Date de publication : 15 décembre 1998

Pagination : 264-268

ISSN : 0246-8743

Référence électronique

"Benoît Habert, Adeline Nazarenko, André Salem, Les linguistiques de corpus », Linx [En ligne], 39 |

1998, mis en ligne le 02 juillet 2012, consulté le 21 septembre 2020. URL : http://

journals.openedition.org/linx/933; DOI : https://doi.org/10.4000/linx.933

Ce document a été généré automatiquement le 21 septembre 2020.

Département de Sciences du langage, Université Paris Ouest 


\section{Benoît Habert, Adeline Nazarenko, André Salem, Les linguistiques de corpus}

Armand Colin, Paris, 1997

\section{RÉFÉRENCE}

Benoît Habert, Adeline Nazarenko, André Salem, Les linguistiques de corpus, Armand Colin, Paris, 1997, 240 pages.

Qu'il s'agisse d'établir dictionnaires et grammaires descriptives, de tester des hypothèses théoriques, d'étudier la variation langagière, l'articulation entre performance et compétence, ou encore de traiter des données pour lesquelles il n'existe pas/plus de locuteur natif (langues anciennes, langue technique ou spécialisée), les corpus électroniques, de plus en plus gigantesques grâce aux progrès de la microinformatique, apparaissent aujourd'hui comme un instrument incontournable.

Dans la première partie de l'ouvrage, les auteurs décrivent les corpus annotés et leurs utilisations, en comparant les intérêts heuristiques, théoriques et pratiques des différentes méthodes d'annotation. Les corpus étiquetés, où des valeurs morphosyntaxiques sont affectées aux unités en fonction de l'objectif de recherche et des moyens humains disponibles, présentent une grande souplesse d'utilisation. S'ils posent le problème du consensus sur le codage, et contraignent bien souvent à un compromis entre la finesse et la productivité du traitement, ils répondent avec une efficacité remarquable à des besoins aussi divers que la typologie de textes ou de locuteurs ou l'étude de l'emploi des parties du discours. Les corpus arborés, eux (illustrés par l'exemple de Suzanne), se heurtent d'emblée au choix théorique entre grammaire des constituants et grammaire de dépendance, en tant qu'analyseurs syntaxiques. Leur performance dépend des objectifs de recherche poursuivis et des langues étudiées. Contrairement aux précédents, dont la mise en œuvre est assez légère, ils nécessitent une collaboration étroite entre linguistes et informaticiens. Les ressources lexicales pour 
l'étiquetage sémantique, qui complètent les outils précédents en permettant la désambiguïsation lexicale, sont en revanche à l'état embryonnaire. Ils sont encore fondés sur des dictionnaires, auxquels s'ajoutent les thesaurus et les bases terminologiques. Cependant, les bases de connaissances conceptuelles (réseaux sémantiques, ontologies) sont en développement, comme le célèbre thesaurus électronique américain Wordnet, formé autour d'un ensemble de synonymes (selon le principe des hiérarchies hyponymiques pour les noms, des classes d'adjectifs avec des pôles antinomiques, et des réseaux de verbes fondés sur l'implication).

3 La deuxième partie de l'ouvrage, intitulée Dimensions transversales, propose d'aller de l'objectif de recherche vers l'outil 'corpus', avec trois axes de recherche particuliers : l'analyse sémantique, les recherches diachroniques et la comparaison de langues à partir des corpus alignés.

4 L'analyse sémantique permet des applications diverses : l'analyse de contenu (stylistique, évolution historique, etc.), repère les thèmes dominants et leur organisation (en littérature, dans le discours politique, sociologique, etc.) ; la recherche documentaire, permet de classer un ensemble de documents avec un net progrès par rapport à la méthode des mots-clés ; l'acquisition de connaissances agit comme une sorte de lecture rapide et automatique du corpus, considéré alors comme une base de connaissances. En matière d'analyse sémantique, synonymie et polysémie constituent les défis majeurs. Les auteurs soulignent que ce domaine manque encore de recul: la méthode y est largement empirique, fondée sur une conception distributionnelle et non logique de la sémantique, prenant d'ailleurs le contre-pied de l'Intelligence Artificielle, du fait des besoins en recherche documentaire.

5 L'utilisation des corpus permet en outre d'étudier les variations en diachronie : c'est le cas du TLF (XIII ${ }^{\text {ème }}$ au XX $\left.{ }^{\text {ème}}\right)$, de Archer (1,7 millions de mots, anglais et américain, de 1650 à nos jours), qui ont permis de renouveler l'analyse de certains faits linguistiques comme l'activation de la productivité de certains préfixes ou suffixes sur le moyen terme, l'évolution de la syntaxe de help sur un longue durée, la position des adjectifs en anglais (qui remet en question les théories admises concernant l'adjectif postposé), l'évolution de l'alternance that/zéro, l'évolution des démonstratifs en français (sur un corpus d'ancien français). Le travail sur ces corpus met à jour des tendances relevant d'abord de certains registres, puis devenant la nouvelle norme. Du fait des difficultés liées aux sources manuscrites et aux nombreuses variantes graphiques, les corpus diachroniques sont plus petits et moins homogènes, souvent plus difficiles à étiqueter, et supposent une analyse manuelle. Ils peuvent cependant répondre au moins en partie à la question de l'acceptabilité d'énoncés en l'absence de locuteurs natifs. Enfin, les corpus en diachronie sont fructueux pour expliquer la morphologie autrement que par la phonétique, ce qui conduit à renouveler les analyses, comme le montre le travail de C. Marchello-Nizia pour l'ancien et moyen français. Les auteurs illustrent abondamment leur propos de problèmes linguistiques concrets, et montrent en quoi la réponse apportée à ce problème par le corpus est originale.

6 Les corpus alignés juxtaposent des textes en deux langues, à la manière de «correspondances traductionnelles». Les corpus diffèrent entre eux principalement par les méthodes d'alignement selon le niveau des unités («résolution»). Selon les auteurs, ces techniques permettent de «récupérer» un certain nombre d'acquis de la traduction automatique restés sans postérité. 
7 La troisième partie de l'ouvrage, intitulée Méthodes et techniques aborde la question des corpus d'un point de vue pratique et matériel.

Une typologie des corpus est proposée (corpus de textes, d'échantillons, de référence, spécialisés, comparables, parallèles). Les enjeux des différents critères de choix des textes sont évalués (volume de données, équilibre des facteurs de variation, sélection en fonction des facilités d'accès). Les auteurs font un point utile sur les nouvelles initiatives en matière de normalisation et de convention (SGML, TEI, etc.), insistent sur la nécessité de documenter un corpus afin d'en permettre une utilisation cohérente (origine du corpus, histoire des sources, convention d'annotation, nécessité d'un 'manuel d'analyse'). Les contraintes institutionnelles, notamment la coopération entre informaticiens et linguistes, et les problèmes juridiques (informatique et liberté, droits d'auteur, protection du corpus), sont également abordés.

9 La question de l'annotation d'un corpus est impossible à aborder de façon exhaustive. Les auteurs choisissent d'en retracer les grands axes et les difficultés (incluant une indication du coût). Les grandes étapes en sont le nettoyage et l'homogénéisation (qui dépend du mode de saisie, et doit tenir compte de la dimension ludique et créative du langage), la segmentation (l'identification des séparateurs, le repérage des traits d'union, des unités complexes, à l'aide de dictionnaires de mots composés comme ceux mis au point par le LADL), l'étiquetage morphosyntaxique (soit par la consultation d'un dictionnaire, soit par analyse morphologique, soit les deux). On a recours pour cela à des règles permettant de déterminer les suites de catégories illicites (impliquant des grammaires locales), ou bien à un fonctionnement par probabilités (pour des langues à ordre des constituants fixe comme anglais ou français) à partir d'un corpus d'apprentissage. Le coût entraîne des compromis comme de choisir des étiquettes basées sur le savoir grammatical courant afin de permettre un traitement et une utilisation par le plus grand nombre. A titre d'exemple, le corpus de Lancaster suppose 39 minutes pour coder 1000 mots. L'analyse syntaxique (parsage) est également fondée soit sur un ensemble de règles positives et négatives, soit sur des probabilités. La performance de tels codages est difficile à évaluer, et de plus, même lorsqu'il est discret, un cadre théorique sous-jacent a priori est nécessaire. Pour le parsage comme pour l'étiquetage morpho-syntaxique, un post-traitement manuel est nécessaire. L'étiquetage sémantique est généralement opéré, lui, sur des unités lexicales hors contexte. On construit des catégories sémantiques en définissant les contextes et les cooccurrences, en calculant la similarité, construisant des classes de mots équivalents selon le point de vue considéré pour parvenir à des réseaux de similarités), puis on projette les catégories sur un corpus.

10 Si les auteurs s'attachent tout au long de ce livre amplement illustré d'exemples à montrer l'intérêt heuristique et l'apport de la linguistique de corpus à la linguistique tout court, en conclusion, ils évoquent néanmoins certaines lacunes. Ils regrettent le faible nombre de ressources encore disponibles pour le français, qu'ils imputent au fossé qui a longtemps existé en France entre la linguistique et le Traitement Automatique du Langage Naturel. Cependant, le travail sur les corpus a selon eux fait sortir le TALN de l'impasse, et ouvre des perspectives nouvelles. Ils soulignent le chemin à parcourir pour une réelle normalisation des corpus, comme la conception de stations de travail textuelles. Ils évoquent le risque d'empirisme absolu qui pèse sur ces méthodes. La linguistique étant une étude de la variation, les linguistiques de corpus 
font naître l'espoir d'un linguistique véritablement expérimentale, à condition d'accepter des ressources qui conservent toujours une part d'approximation.

11 Le mérite de cet ouvrage est de décrire par le menu, dans un langage accessible à tous, et d'un point de vue de linguiste, des méthodes qui, peut-on l'espérer, ont cessé d'être à la fois méconnues et diabolisées par les linguistes et surestimées par les inconditionnels. Alors que certaines illusions reculent (comme l'annotation automatique complète), l'analyse de corpus, comme méthode dépassant les clivages théoriques, apparait comme un outil prometteur.

12 Un seul bémol peut-être à cet ouvrage constructif, amplement documenté, et toujours attentif aux questions théoriques : le parti pris d'un plan très didactique provoque souvent un sentiment de redondance. L'écriture à trois plumes n'y est peut être pas étrangère. 